\title{
Chemical-Induced Unfolding of Cofactor-Free Protein Monitored by Electrochemistry
}

\author{
Liang-Hong Guo* and Na Qu \\ State Key Laboratory of Environmental Chemistry and Ecotoxicology, Research Center for Eco-environmental Sciences, \\ Chinese Academy of Sciences, P.O. Box 2871, 18 Shuangqing Road, Beijing 100085, China
}

Protein folding has been studied extensively with an aim to better understanding of the relationship between protein sequence, structure, and function. A large variety of techniques have been developed and utilized to probe protein conformation and folding/unfolding transition. In this report, electrochemical monitoring of urea-induced unfolding of a large cofactor-free protein, bovine serum albumin (BSA), is described. Enhanced electrochemical oxidation of tyrosine and tryptophan in free amino acids and in BSA was achieved on an indium tin oxide electrode by using an electron mediator, $0 \mathrm{~s}(\mathrm{bpy})_{2} \mathrm{dppz}\left(\mathrm{bpy}=2,2^{\prime}\right.$ bipyridine, $\operatorname{dppz}=$ dipyrido[3,2-a:2',3'-c]phenazine). The oxidation current was used as a signal reporter in the monitoring of urea-induced BSA denaturation. At high urea concentrations, the electrochemical signal increased by 3 -fold relative to the native protein. The increase is attributed to the closer contact between the oxidizable residues in the unfolded $\mathrm{BSA}$ and $\mathrm{Os}(\mathrm{bpy})_{2} \mathrm{dppz}$. The degree of unfolding assessed by electrochemistry correlates well with the established fluorescence technique in the range of $0-10 \mathrm{M}$ urea. The method can be used to investigate the unfolding process of other cofactor-free proteins.

Protein folding has been studied extensively in the past two decades or so with an aim to better understanding the relationship between protein sequence, structure, and function., ${ }^{1,2} \mathrm{~A}$ large variety of techniques have been developed and utilized to probe protein conformation and folding/unfolding transition, such as mass spectrometry, fluorescence, calorimetry, circular dichroism, IR/Raman spectroscopy, and NMR. ${ }^{3}$ Among them, fluorescence has become a popular tool due to its simple procedure and high sensitivity. ${ }^{4,5}$ In particular, the intrinsic fluorescence of the tryptophan residue allows for label-free monitoring of protein confor-

* Corresponding author. Phone/Fax: 86-10-62849685. E-mail: LHGuo@ rcees.ac.cn.

(1) Kim, P. S.; Baldwin, R. L. Annu. Rev. Biochem. 1990, 59, 631.

(2) Matthews, C. R. Annu. Rev. Biochem. 1993, 62, 653.

(3) Righetti, P. G.; Verzola, B. Electrophoresis 2001, 22, 2359. and references therein.

(4) Pace, C. N.; Laurents, D. V.; Thomson, J. A. Biochemistry 1990, 29, 2564

(5) van Nuland, N. A. J.; Chiti, F.; Taddei, N.; Raugei, G.; Ramponi, G.; Dobson, C. M. J. Mol. Biol. 1998, 283, 883. mation change. Due to the low instrument cost, adequate sensitivity, and easy operation, electrochemistry has also attracted considerable interest among researchers. A unique feature of electrochemistry is that both kinetic and thermodynamic information of the redox process can be extracted from the popular cyclic voltammogram using simple protocols. As such, redox-statedependent refolding of guanidine-denatured cytochrome $c$ was investigated by monitoring the direct electrochemical response of the iron-porphyrin unit inside the protein. ${ }^{6-8}$ Information about the transition and thermodynamics of the process was obtained in a few voltage scans. Obviously, the utility of this approach is limited to proteins with redox-active cofactors.

Many proteins contain tyrosine and/or tryptophan residues that can be oxidized. Since these residues are usually located in the interior of a native protein and become more exposed when the protein unfolds, they can be used as a signal reporter for the conformational state of the protein. Direct electrochemical oxidation of free tyrosine and tryptophan amino acids was obtained on carbon electrodes and was utilized in the quantitative analysis. ${ }^{9-13}$ Small peptides containing the two amino acids were analyzed by detecting their oxidation current. ${ }^{14}$ Very recently, aggregation of amyloid $\beta$ peptides (a 4-kDa molecular marker for Alzheimer's disease) was detected by measuring the oxidation signal of tyrosine on glassy carbon electrode. ${ }^{15}$ However, the adsorption/ desorption process of large proteins on carbon and metal surfaces was found to dominate their electrochemical response and render the conformation study impractical. ${ }^{16,17}$

We report here the first electrochemical monitoring of urea-induced unfolding of a large cofactor-free protein, bovine serum albumin (BSA). Electrochemical oxidation of tyrosine

(6) Bixler, J.; Bakker, G.; McLendon, G. J. Am. Chem. Soc. 1992, 114, 6938

(7) Ferri, T.; Poscia, A.; Ascoli, F.; Santucci, R. Biochim. Biophys. Acta 1996, 1298, 102.

(8) Zhu, Y.; Dong, S. Bioelectrochem. Bioenerg. 1996, 41, 107.

(9) Malfoy, B.; Reynaud, J. A. J. Electroanal. Chem. 1980, 114, 213.

(10) Brabec, V.; Mornstein, V. Biophys. Chem. 1980, 12, 159.

(11) Wang, J.; Chen, G. Talanta 2003, 60, 1239.

(12) Jin, J.-P.; Lin, X.-Q. Electrochem. Comm. 2004, 6, 454

(13) Xu, Q.; Wang, S.-F. Microchim. Acta 2005, 151, 47.

(14) Cai, X.; Rivas, G.; Farias, P. A. M.; Shiraishi, H.; Wang, J.; Palecek, E. Anal. Chim. Acta. 1996, 332, 49.

(15) Westergaard, M.; Kermna, K.; Saito, M.; Nagatani, N.; Takamura, Y.; Tamiya, E. J. Am. Chem. Soc. 2005, 127, 11892

(16) Reynaud, J. A.; Malfoy, B.; Bere, A. Bioelectrochem. Bioenerg. 1980, 7, 595

(17) Brabec, V.; Schindlerova, I. Bioelectrochem. Bioenerg. 1981, 8, 451. 
and tryptophan in BSA was catalyzed by an electron mediator,

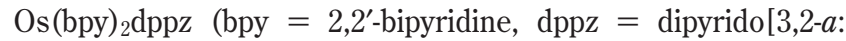
$\left.2^{\prime}, 3^{\prime}-c\right]$ phenazine). As BSA unfolds in the presence of urea, accessibility of the metal complex to the oxidizable amino acid residues buried inside the native protein is increased, resulting in enhanced catalytic current. The degree of unfolding assessed by electrochemistry correlates well with the established fluorescence technique. The method can be used to investigate the unfolding process of other cofactor-free proteins. In principle, it can also be employed in the study of biological binding reactions in which protein conformational change is involved.

\section{EXPERIMENTAL SECTION}

Bovine serum albumin (fraction V) and urea (USP grade) were purchased from MP Biochemicals and Amresco, respectively. The amino acids (tyrosine, tryptophan, cystine, methionine, and histidine) were obtained from Shanghai Biochemicals (Shanghai, China). BSA was denatured in urea by overnight (15 h) incubation. $\mathrm{Os}(\mathrm{bpy})_{2}(\mathrm{dppz}) \mathrm{Cl}_{2}$ was a kind gift from Dr. Dominic Hull in Prof. H. Holden Thorp's group at the University of North Carolina. It was prepared according to the published procedures ${ }^{18,19}$ and characterized by UV-visible spectrophotometry, cyclic voltammetry, and elemental analysis.

Indium tin oxide (ITO) conductive glass was obtained from WeiGuang Corp. (Shenzhen, Guangdong Province, P. R. China) and cut into size $2.5 \times 0.5 \mathrm{~cm}$ electrodes. The electrodes were cleaned in an ultrasonic cleaner sequentially with each of the following solutions: household detergent in water (15 min), deionized water (2 min, twice), acetone (5 min), 2-propanol (5 $\mathrm{min}$ ), and deionized water (10 min, twice). Cyclic voltammetry was performed on a CHI 660B electrochemistry analyzer (Austin, TX) with a $\mathrm{Pt}$ counter electrode and an $\mathrm{Ag} / \mathrm{AgCl}$ reference electrode. The position of the ITO electrode in the electrochemical cell was secured with a Teflon holder so that its area in contact with the electrolyte was fixed $\left(0.5 \mathrm{~cm}^{2}\right)$.

Fluorescence emission spectra were measured on a PerkinElmer LS55 luminescence spectrometer.

\section{RESULTS AND DISCUSSION}

BSA was chosen in our study as a model protein to demonstrate electrochemical detection of chemical-induced conformational change of cofactor-free proteins. It is a large single polypeptide ( $\mathrm{MW} \sim 65000$ ) with 21 tyrosine, 3 tryptophan, 35 cysteine, 5 methionine, and 17 histidine residues that can potentially be oxidized. ${ }^{20}$ An ITO electrode was employed in the study because the surface is relatively hydrophilic and chemically very inert. Therefore, there is less tendency for large biomolecules to adsorb on the surface and complicate the situation, ${ }^{21,22}$ as is the case on metal and carbon electrodes. However, as illustrated later, the oxidation current of BSA on ITO is low even with such a large number of oxidizable residues. To increase the signal, an electrochemically reversible metal complex, Os(bpy) ${ }_{2} \mathrm{dpp} z$, was employed as a redox mediator to shuttle electrons between the

(18) Leone, A. M.; Hull, D. O.; Wang, W.; Thorp, H. H.; Murray, R. W. J. Phys. Chem. A 2004, 108, 9787.

(19) Welch, T. W.; Corbett, A. H.; Thorp, H. H. J. Phys. Chem. 1995, 99, 11757.

(20) Brown, J. R. In Albumin Structure, Function and Uses; Rosenoer, V. M., Oratz, M., Rothschild, M. A., Eds.; Pergamon: Oxford, 1977; pp 27-51.

(21) Yeh, P.; Kuwana, T. Chem. Lett. 1977, 1145.

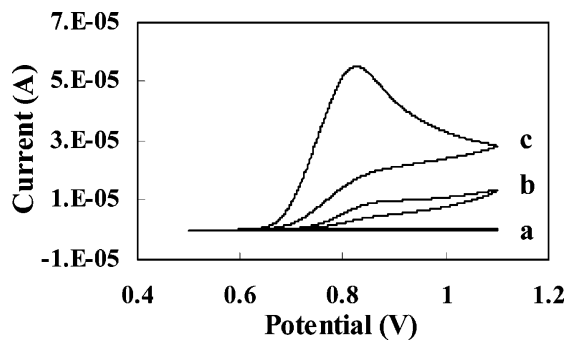

Figure 1. Cyclic voltammograms of (a) $5 \mu \mathrm{M}$ Os(bpy) $)_{2} \mathrm{dppz}$, (b) $2 \mathrm{mM}$ tyrosine, and (c) $5 \mu \mathrm{M}$ Os(bpy) ${ }_{2} \mathrm{dppz}$ and $2 \mathrm{mM}$ tyrosine. Working electrode: indium tin oxide. Reference: $3 \mathrm{M} \mathrm{Ag} / \mathrm{AgCl}$. Supporting electrolyte: $100 \mathrm{mM}$ sodium phosphate, $\mathrm{pH}$ 7.3. Scan rate: $30 \mathrm{mV} / \mathrm{s}$.

amino acid residues and the electrode. Many metal complexes have been employed previously as electron mediators to establish efficient electrical communication between redox enzymes and the electrode. ${ }^{23-25}$ The best known example is ferrocene, which is currently used in the commercial glucose meter. ${ }^{26}$ Ruthenium polypyridine complexes have also been used to catalyze the oxidation of DNA bases on ITO. ${ }^{27,28}$

The standard potential of $\mathrm{Os}(\mathrm{bpy})_{2} \mathrm{dpp} z$ is $0.72 \mathrm{~V}$ versus $\mathrm{Ag} / \mathrm{AgCl},{ }^{19}$ high enough to oxidize tyrosine $(0.55 \mathrm{~V})$ and tryptophan $(0.7 \mathrm{~V}) .{ }^{14}$ This metal ligand has been found to be a DNA intercalator and has been employed as a signal indicator in DNA detection. ${ }^{19}$ It was selected in the present work to serve as a redox mediator solely due to its electrochemical reversibility and suitable standard potential. To test the approach of electron mediation, electrochemical oxidation of the free amino acids was first investigated with the mediator. As shown in Figure 1, tyrosine itself produced a low and irreversible oxidation wave on ITO electrode. Upon addition of $5 \mu \mathrm{M}$ Os(bpy $)_{2} \mathrm{dpp} z$, the current increased by almost 7-fold (oxidation current of the metal complex is comparatively low). The metal complex itself exhibited reversible, diffusion-controlled voltammetric response on ITO electrodes, as indicated by the linear relationship between the peak current and square root of the scan rate (Figure S1 in Supporting Information). From the cyclic voltammogram, the second-order rate constant of the redox reaction between tyrosine and $\mathrm{Os}(\mathrm{bpy}){ }_{2} \mathrm{dpp} z$ is calculated ${ }^{29}$ to be $2.0( \pm 0.3) \times 10^{3} \mathrm{M}^{-1} \mathrm{~s}^{-1}$. Catalyzed current for tryptophan was also observed, although to a less extent (Figure 2). As discussed later, because the number of tyrosine residues in BSA is much greater than tryptophan, contribution from the latter to the catalyzed oxidation current of BSA can be neglected. Catalyzed current of histidine, methionine, and cystine is very low in comparison with tyrosine (see Supporting Information). The sluggish response of these amino acids in the absence of $\mathrm{Os}(\mathrm{bpy})_{2} \mathrm{dppz}$ is consistent with the general observation that the electrochemistry of most organic molecules

(22) Armistead, P. M.; Thorp, H. H. Anal. Chem. 2000, 72, 3764.

(23) Hill, H. A. O.; Sanghera, G. S. In Biosensors: A Practical Approach; Cass, A. E. G., Ed.; Oxford University Press: London, 1990; pp 19-46.

(24) Chaubey, A.; Malhotra, B. D. Biosens. Bioelectron 2002, 17, 441.

(25) Degani, Y.; Heller, A. J. Am. Chem. Soc. 1989, 111, 2357.

(26) Cass, A. E. G.; Davis, G.; Francis, G. D.; Hill, H. A. O.; Aston, W. J.; Higgins, I. J.; Plotkin, E. V.; Scott, L. D. L.; Turner, A. P. F. Anal. Chem. 1984, 56, 667.

(27) Thorp, H. H. Top. Curr. Chem. 2004, 237, 159.

(28) Rusling, J. F. Biosens Bioelectron. 2004, 20, 1022.

(29) Nicholson, R. S.; Shain, I. Anal. Chem. 1964, 36, 706. 


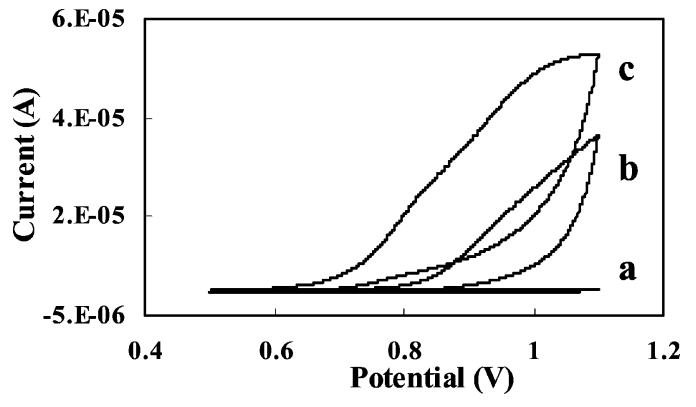

Figure 2. Cyclic voltammograms of (a) $5 \mu \mathrm{M}$ Os(bpy) ${ }_{2} \mathrm{dppz}$, (b) 2 $\mathrm{mM}$ tryptophan, and (c) $5 \mu \mathrm{M}$ Os(bpy) ${ }_{2} \mathrm{dppz}$ and $2 \mathrm{mM}$ tryptophan. Working electrode: indium tin oxide. Reference: $3 \mathrm{M} \mathrm{Ag} / \mathrm{AgCl}$. Supporting electrolyte: $100 \mathrm{mM}$ sodium phosphate, $\mathrm{pH}$ 7.3. Scan rate: $30 \mathrm{mV} / \mathrm{s}$.

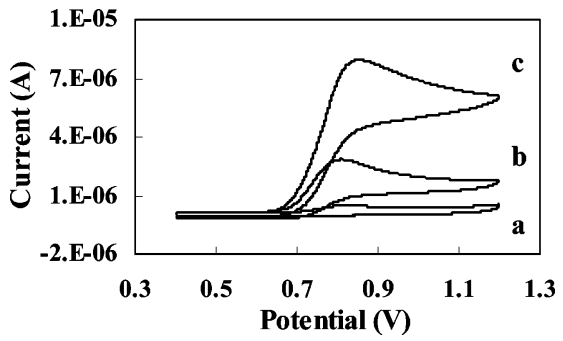

Figure 3. Cyclic voltammograms of (a) $0.1 \mathrm{mM} B S A$, (b) after addition of $5 \mu \mathrm{M}$ Os(bpy $)_{2} \mathrm{dppz}$, and (c) after addition of $5 \mu \mathrm{M}$ Os(bpy) $)_{2} \mathrm{dppz}$ and $10 \mathrm{M}$ urea. Working electrode: indium tin oxide. Reference: $3 \mathrm{M} \mathrm{Ag} / \mathrm{AgCl}$. Supporting electrolyte: $100 \mathrm{mM}$ sodium phosphate, $\mathrm{pH}$ 7.3. Scan rate: $30 \mathrm{mV} / \mathrm{s}$.

such as amines, ${ }^{30}$ organic acids, ${ }^{31}$ and DNA bases ${ }^{22}$ on ITO is slow. More facile reaction was observed on metal electrodes but was complicated by adsorption process. ${ }^{9,32}$

The Os complex is also an effective electron mediator for BSA. The protein displayed very low oxidation current on ITO electrode, as shown in Figure 3. Since there is no redox-active cofactor in the protein, the signal is produced by the oxidation of tyrosine and tryptophan residues. Similar to the free amino acids, the current was enhanced by 7 -fold after addition of $\mathrm{Os}(\mathrm{bpy})_{2} \mathrm{dppz}$ into the protein solution. The response can be attributed mostly to the oxidation of tyrosine residues in the protein due to its large number (21 residues) and high reactivity (Figure 1). The calculated rate constant for the redox reaction between BSA and Os (bpy) $)_{2} \mathrm{dppz}$ is $12( \pm 0.2) \mathrm{M}^{-1} \mathrm{~s}^{-1}$. This rate is significantly lower than the one with free tyrosine, reflecting the hindrance of the protein matrix to the diffusion of a relatively large-sized mediator. The lower rate is also manifested by the substantially lower current of BSA, even though tyrosine concentration in the protein $(21 \times$ $0.1 \mathrm{mM})$ is about the same as the free amino acid $(2 \mathrm{mM})$. Figure 3 also shows that, after BSA was denatured with $10 \mathrm{M}$ urea, its oxidation signal increased by 3 -fold relative to the native protein (electrochemical response of Os(bpy) $)_{2} \mathrm{dpp} z$ was reversible even in $10 \mathrm{M}$ urea/100 $\mathrm{mM}$ sodium phosphate, $\mathrm{pH}$ 7.3). As stated above, the side chains of both tyrosine and tryptophan are

(30) Zheng, D.; Wang, N.; Wang, F.-Q.; Dong, D.; Li, Y.-G.; Yang, X.-Q.; Guo, L.-H.; Cheng, J. Anal. Chim. Acta 2004, 508, 225.

(31) Kanoufi, F.; Bard, A. J. J. Phys. Chem. B 1999, 103, 10469.

(32) Reynaud, J. A.; Malfoy, B.; Canesson, P. J. Electroanal. Chem. 1980, 114, 195.
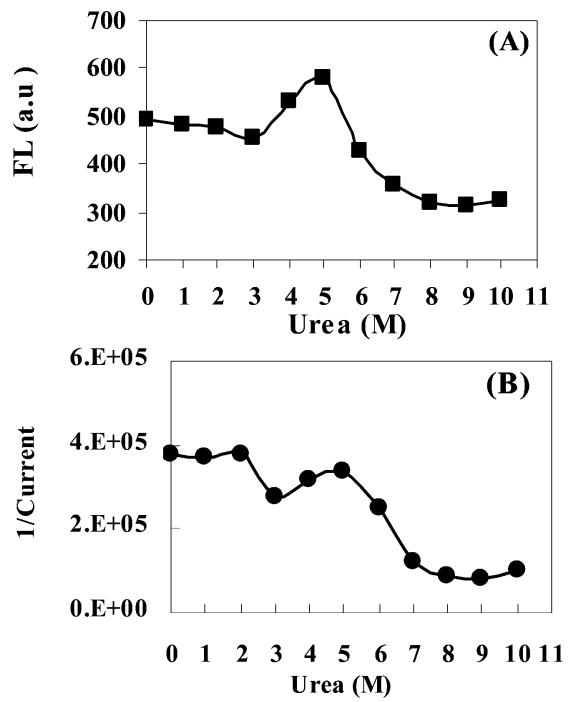

Figure 4. (A) Intrinsic fluorescence emission intensity of $0.1 \mathrm{mM}$ BSA in $100 \mathrm{mM}$ sodium phosphate, $\mathrm{pH} 7.3$, as a function of urea concentration. Excitation wavelength: $280 \mathrm{~nm}$. Emission wavelength: $290-500 \mathrm{~nm}$. (B) Reciprocal of the oxidation current of 0.1 $\mathrm{mM}$ BSA catalyzed with $5 \mu \mathrm{M}$ Os(bpy $)_{2} \mathrm{dppz}$ as a function of urea concentration. The current is corrected for slowed diffusion in urea. Working electrode: indium tin oxide. Reference: $3 \mathrm{M} \mathrm{Ag} / \mathrm{AgCl}$. Supporting electrolyte: $100 \mathrm{mM}$ sodium phosphate, $\mathrm{pH}$ 7.3. Scan rate: $30 \mathrm{mV} / \mathrm{s}$.

nonpolar and are usually located in the interior of the water-soluble protein. They become more exposed when the protein unfolds in the presence of urea and, consequently, more accessible to the electron mediator. The calculated rate constant is $106( \pm 15) \mathrm{M}^{-1}$ $\mathrm{s}^{-1}$ for the denatured protein.

Urea-induced unfolding of BSA has been studied previously by monitoring the change of its intrinsic fluorescence. ${ }^{33}$ As the protein unfolds, the emission intensity of tryptophan residues decreases, and the peak position shifts to a longer wavelength. To validate our new method, urea-induced denaturation of BSA was monitored by both fluorescence and electrochemistry. Figure $4 \mathrm{~A}$ shows the change of fluorescence intensity as a function of urea concentration, which follows the general trend described in the literature. Unlike fluorescence, the electrochemical signal increased at high urea concentrations (as shown in Figure 3). To make a better comparison between fluorescence and electrochemistry, the reciprocal of the oxidation current is used as an indicator for the state of protein unfolding and is plotted in Figure 4B. It is worth noting that, as urea concentration in the electrolyte was increased, the solution became more viscous. As a result, the voltammetric response of Os(bpy $)_{2} \mathrm{dppz}$ alone decreased due to slower diffusion (Figure S5 in Supporting Information). Therefore, the catalytic current of BSA is corrected for the slowed diffusion using the data in Figure 5S so that the conformational change of the protein is the sole factor. The similarity (correlation coefficient 0.92 ) of the pattern displayed in the two denaturation curves suggests that catalytic electrochemistry can be employed as a new tool for the study of the unfolding process of cofactor-free proteins. The correspondence between electrochemistry and fluorescence observed in our study is not neces-

(33) Khan, M. K.; Agarwal, S. K.; Hangloo, S. J. Biochem. 1987, 102, 313. 
sarily going to be the case for BSA unfolded in other denaturants or for other proteins. While fluorescence responds to the change of local polarity in the protein, catalytic voltammetry depends on the permeability of the electron mediator inside the protein. Therefore, there might be cases where fluorescence changes due to slight structural perturbation that electrochemistry does not respond.

In conclusion, urea-induced unfolding of BSA was monitored by catalytic electrochemistry on an indium tin oxide electrode. Enhanced electrochemical signal of the protein was obtained using Os (bpy) $)_{2} \mathrm{dpp} z$ as an electron mediator for the oxidation of tyrosine and tryptophan residues located in the interior of the protein. The denaturation process monitored by the new method correlates well with the established fluorescence technique. The approach can be extended to the investigation of other cofactor-free proteins.

\section{ACKNOWLEDGMENT}

This work was supported by a start-up fund provided by the Chinese Academy of Sciences. Os (bpy) ${ }_{2} \mathrm{dppz}$ was a kind gift from Dr. Dominic Hull in Prof. H. Holden Thorp's group at the University of North Carolina. We thank the reviewers for constructive comments and suggestions.

\section{SUPPORTING INFORMATION AVAILABLE}

Cyclic voltammetry and fluorescence data. This material is available free of charge via the Internet at http://pubs.acs.org.

Received for review February 24, 2006. Accepted July 25, 2006.

AC060351H 